\title{
DEPREDACIÓN DE LA LAGARTIJA PLESTIODON COPEI (SCINCIDAE) POR LA RANA LITHOBATES MONTEZUMAE (RANIDAE) EN MICHOACÁN, MÉXICO PREDATION OF THE LIZARD PLESTIODON COPEI (SCINCIDAE) BY LITHOBATES MONTEZUMAE (RANIDAE) IN MICHOACÁN, MÉXICO
}

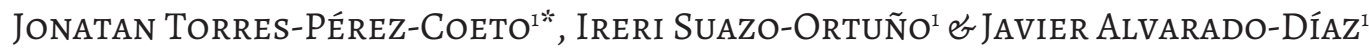 \\ ${ }^{1}$ Laboratorio de Herpetología, Departamento de Zoología, Instituto de Investigaciones sobre los Recursos Naturales, Universidad Michoacana de San \\ Nicolás de Hidalgo. Av. San Juanito Itzicuaro s/n. Col. Nueva Esperanza C.P. 58337 Morelia, Michoacán, México. \\ *Correspondencia: crotalus365@gmail.com
}

Abstract.- An unusual observation of predation of the Cope's Skink Lizard, Plestiodon copei, by the Montezuma Leopard Frog, Lithobates montezumae, is reported.

Keywords.- Predation, Plestiodon copei, Michoacán.

Resumen.- En esta nota se reporta un caso inusual de depredación de la lagartija Lincer de Cope Plestiodon copei, por la Lithobates de Moctezuma Lithobates montezumae.

Palabras clave.- Depredación, Plestiodon copei, Michoacán.

La lagartija Lincer de Cope Plestiodon copei, Taylor 1933 o salamanquesa como es coloquialmente conocida es endémica a México. Presenta una amplia distribución en las zonas montañosas de los estados de México, Michoacán, Morelos, Puebla, Veracruz y Ciudad de México (Smith \& Smith 1976; Ramírez-Bautista et al., 2009). Es una especie de cuerpo alargado y delgado, de tamaño mediano. Las hembras son ligeramente más grandes y robustas que los machos; la cabeza es puntiaguda y las extremidades son marcadamente cortas y robustas. Las escamas del cuerpo son pequeñas, lisas, cicloides y brillantes. Presenta una franja de color café metálico claro que abarca la región dorsal del cuerpo y cabeza; con dos líneas blancas laterales que se originan en la punta del hocico y terminan en la base de la cola. La región ventral del cuerpo es color gris, y la cola se presenta un tono azul metálico intenso en machos o ligeramente azulado en hembras.

Esta especie habita parches de bosques templados en las partes más altas de la Faja Volcánica Transmexicana (Uribe-Peña et al., 1999). Es de hábitos principalmente diurnos y terrestres. Es frecuente encontrarla entre troncos secos o entre la hojarasca y bajo rocas. Se alimenta de insectos y es vivípara (Uribe-Peña et al., 1999). A las 10:00 h del 14 Julio 2019 mientras realizábamos un monitoreo de Ambystoma ordinarium en el cauce del río Puente de Vacas a $8 \mathrm{~km}$ al sur de Agostitlán, municipio de Ciudad Hidalgo (19 $31^{\prime} 10.00^{\prime \prime} \mathrm{N}$ y 100 $37^{\prime} 1.91^{\prime \prime}$ O Datum: WGS 84, $\left.2446 \mathrm{msnm}\right)$,
Michoacán, se observó una serpiente (Thamnophis cyrtopsis) en el cauce del arroyo y un ejemplar de Plestiodon copei (CHUM-1850, $\mathrm{LHC}=50 \mathrm{~mm}$ y $\mathrm{LC}=61 \mathrm{~mm}$ ) en la ribera del río sobre pasto. $\mathrm{Al}$ moverse, la lagartija fue capturada de un salto por un ejemplar adulto de Lithobates montezumae (CHUM-1852, LHC $=78 \mathrm{~mm}$ ), que estaba presumiblemente al acecho. Posteriormente, se procedió a colectar y fotografiar el ejemplar de L. montezumae. Esta fue transportada al laboratorio donde fue fijada y se realizó la disección del estómago para corroborar la presencia del ejemplar de P. copei (Figs. 1 y 2). Ambos ejemplares fueron depositados en la Colección Herpetológica de la Universidad Michoacana (CHUM). Durante la disección, también se encontraron algunos ejemplares de escarabajos (Insecta: Scarabeidae). Los muestreos se ampararon en el permiso de colecta $\mathrm{N}^{\circ} \mathrm{SGPA} / \mathrm{DGVS} / 001450$ /18. La depredación de lagartijas por anuros es relativamente común (Ribeiro \& Freire, 2009) aunque algunas lagartijas como las que pertenecen al género Plestiodon se consideran rápidas para ser atrapadas por Lithobates (Price-Rees et al., 2010).

A nuestro conocimiento este es el primer registro de la depredación de P. copei por una Lithobates o por la L. montezumae.

Agradecimientos.- Se agradece a Luis Canseco-Márquez por la corroboración de la identidad de las especies $P$. copei y $L$. montezumae y al proyecto de ciencia básica de Conacyt No del proyecto CB-2015-01-259173 de Ciencia Básica que financió el 


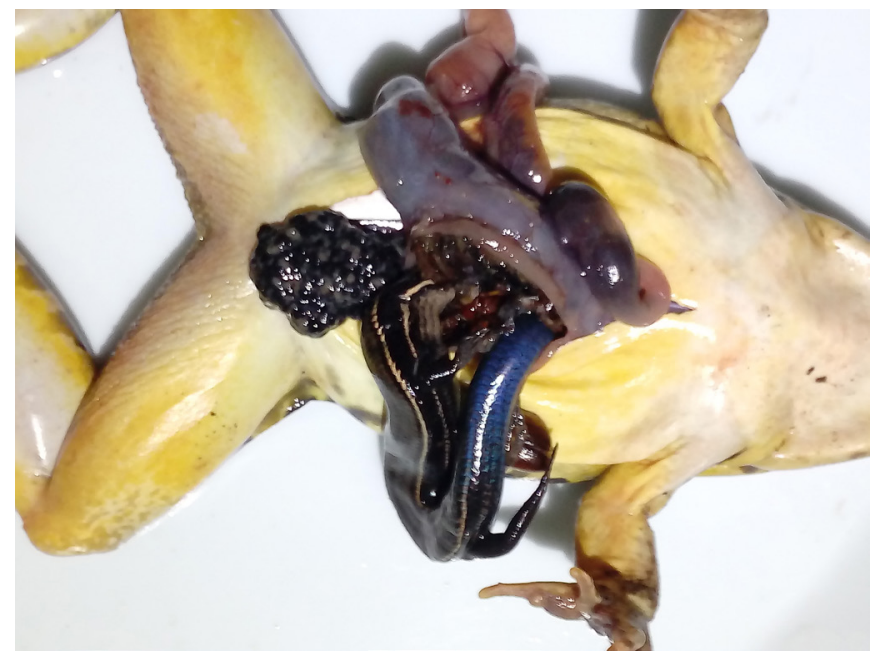

Figure 1. Dissection of $L$. montezumae showing the predated specimen of $P$. copei. Picture: JTPC.

Figura 1. Disección de L. montezumae mostrando el ejemplar de P. copei depredado. Fotografía: JTPC.

trabajo de campo para el monitoreo de Ambystoma ordinarium, debido al cuál se registró de manera incidental la observación referida en la nota.

\section{LITERATURA CITADA}

Price-Rees, S.J., G.P. Brown \& R. Shine. 2010. Predation on toxic cane toads (Bufo marinus) may imperil bluetongue lizards (Tiliqua scincoides intermedia, Scincidae) in tropical Australia. Wildlife Research. 37:166-173.

Ramírez-Bautista, A., U. Hernández-Salinas, U. García-Vázquez, A. Leyte-Manrique \& L. Canseco-Márquez. 2009. Herpetofauna del Valle de México: Diversidad y conservación. Universidad

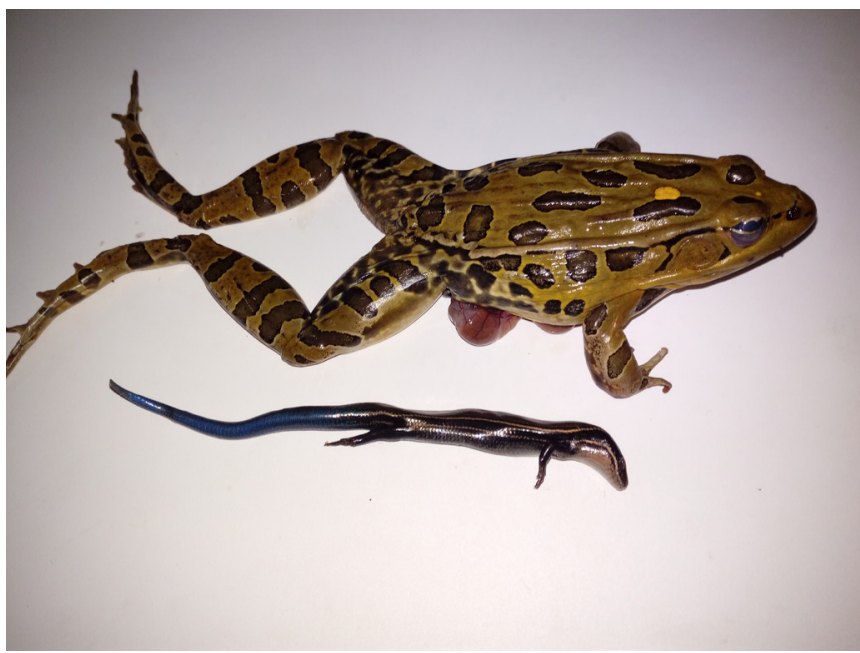

Figure 2. Dorsal view of $L$. montezumae and lateral view of the specimen of $P$. copei predated by the former.

Figura 2. Vista dorsal de L. montezumae y lateral del ejemplar de P. copei depredado por ésta.

Autónoma del Estado de Hidalgo. Comisión Nacional para El Conocimiento y Uso de la Biodiversidad. México. 213 pp.

Ribeiro, L.B. \& E.M.X. Freire. 2009. Tropidurus hispidus. Frog predation. Herpetological Review. 40:228.

Smith, H.M. \& R.B. Smith. 1976. Synopsis of the herpetofauna of México Vol. III (Source analysis and index for Mexican reptiles) J. Johnson, U.S.A.

Uribe-Peña, Z., A. Ramírez-Bautista \& G. Casas. 1999. Anfibios y reptiles de las Serranías del distrito Federal, México. Universidad Nacional Autónoma de México. Cuadernos del Instituto de Biología 32:1-119. 